\title{
REMARKS ON THE AHLFORS CLASS $N$ IN AN ANNULUS
}

\author{
W. CIEŚLAK and J. ZAJĄC
}

\section{Introduction}

In [1] Ahlfors investigated the class $N$ of complex-valued $L^{\infty}$ functions $v$ in the unit disc for which the antilinear part of variation of normalized quasiconformal mappings vanishes, where the mappings are generated by complex dilatation of the form $t v, t$ being a real parameter. He gave two important characterizations of this class and its explicit form with the help of an analytic function in the unit disc. This class $N$ has also been investigated by Reich and Strebel [4] in connection with Teichmüller mappings. Very deep investigation of the class $N$ has been conducted by Reich [3]. He has also considered the class $N$ in an annulus with "inward extension".

In this paper we shall consider the class $N$ in an annulus without any other restrictions. The results presented here are a continuation of the theory published in [3], [4] and [6].

As it has been shown by many authors (cf. [2]), the class $N$ plays a very important role in some investigations of extremal problems within the class of quasiconformal mappings in the unit disc and in an annulus as well with the help of parametrical methods.

\section{The class $N_{r}$}

Let $\mu$ be a complex-valued measurable function in an annulus $\Delta_{r}=\{z: r \leqq|z| \leqq 1\}$, $0 \leqq r<1$, satisfying the condition

$$
\|\mu\|_{\infty}=\inf _{E} \sup _{z \in \Delta_{r} \backslash E}|\mu(z)|<1,
$$

where the infimum is taken over all sets of the plane measure zero. It is well known that there exists exactly one number $R, 0 \leqq R<1$, and one $Q$-quasiconformal mapping $f$ of the annulus $\Delta_{r}$ onto $\Delta_{R}$ satisfying the Beltrami equation

$$
f_{\bar{z}}=\mu f_{z} \quad \text { with } \quad f(1)=1,
$$

where $Q=\left(1+\|\mu\|_{\infty}\right) /\left(1-\|\mu\|_{\infty}\right)$. 
Suppose now that $\mu=t v$, where $\|v\|_{\infty}<\infty$, and $0 \leqq t<1 /\|v\|_{\infty}$. Denote explicitly the dependence of $f$ on $v: f(z, t)=f[v](z, t), r \leqq|z| \leqq 1$. Let

$$
f[v](z)=\lim _{t \rightarrow 0} \frac{1}{t}\{f[v](z, t)-z\} .
$$

This expression is well defined and depends linearly on $v$ (cf. [1]). From $f[v]_{\bar{z}}=t v f[v]_{z}$ it follows that

$$
f[v]_{\bar{z}}=v .
$$

It is well known that (3) is satisfied only if

$$
\dot{f}[v](\zeta)=-\frac{1}{\pi} \iint_{\Delta_{r}} \frac{v(z)}{z-\zeta} d x d y-F(\zeta)
$$

with holomorphic $F$.

Thus we have (cf. [2])

$$
\begin{aligned}
f[v](\zeta)= & \frac{\zeta}{2 \pi} \iint_{\Delta_{r}} \sum_{k=-\infty}^{+\infty}\left[\frac{v(z)}{z^{2}}\left(\frac{\zeta+r^{2 k} z}{\zeta-r^{2 k} z}-\frac{1+r^{2 k} z}{1-r^{2 k} z}\right)\right. \\
& \left.-\frac{\overline{v(z)}}{z^{2}}\left(\frac{1+r^{2 k} \zeta \bar{z}}{1-r^{2 k} \zeta \bar{z}}-\frac{1+r^{2 k} \bar{z}}{1-r^{2 k} \bar{z}}\right)\right] d x d y .
\end{aligned}
$$

We see that $f$ is a linear continuous operator which maps every $v \in L^{\infty}\left(\Delta_{r}\right)$ on a function $f[v]$. As it is shown in [2], the relations $|f[v](z, t)|=1$ for $|z|=1$ and $|f[v](z, t)|=R[v](t)$ for $|z|=r$ yield

$$
\operatorname{Re}\{\bar{z} \hat{f}[v](z)\}=\left\{\begin{array}{lll}
0 & \text { for } & |z|=1, \\
r \varrho & \text { for } & |z|=r
\end{array}\right.
$$

where $\varrho=\lim _{t \rightarrow 0} \frac{1}{t}\{R[v](t)-r\}$. Analogically we can verify that

$$
\operatorname{Re}\{\bar{z} f[i v](z)\}=\left\{\begin{array}{lll}
0 & \text { for } & |z|=1 \\
r Q^{*} & \text { for } & |z|=r
\end{array}\right.
$$

where $\varrho^{*}=\lim _{t \rightarrow 0} \frac{1}{t}\{R[i v](t)-r\}$. For more details see [2].

We recall [2] that

$$
\varrho=\frac{r}{2 \pi} \iint_{\Delta_{r}}\left[\frac{v(z)}{z^{2}}+\frac{\overline{v(z)}}{\bar{z}^{2}}\right] d x d y,
$$

by which

$$
\varrho^{*}=\frac{i r}{2 \pi} \iint_{\Delta_{r}}\left[\frac{v(z)}{z^{2}}-\frac{\overline{v(z)}}{\bar{z}^{2}}\right] d x d y .
$$


Following Ahlfors [1] let us decompose the variation $f[v]$ defined by (2) as follows:

$$
f[v]=\frac{1}{2}\{f[v]+i f[i v]\}+\frac{1}{2}\{f[v]-i f[i v]\},
$$

where the first part is antilinear and the second one is linear with respect to the complex multipliers. By the definition of $\dot{f}[v]$ we can see that $\{\dot{f}[v]+i \dot{f}[i v]\}_{\bar{z}}=0$, i.e.,

$$
\Phi[v]=f[v]+i f[i v]
$$

is always a holomorphic function. The antilinearity is expressed by $\Phi[i v]=-i \Phi[v]$.

We denote by $N_{r}$ the subspace of $L^{\infty}\left(\Delta_{r}\right)$ which is formed by all $v$ with $\Phi[v]=0$. It is a complex linear subspace of $L^{\infty}\left(\Delta_{r}\right)$. Now we can state

Theorem 1. An element $v$ of $L^{\infty}\left(\Delta_{r}\right)$ belongs to $N_{r}$ if and only if one of the following assumptions holds:

$$
\begin{gathered}
f[v](\zeta)=\left\{\begin{array}{lll}
0 & \text { for } & |\zeta|=1, \\
\frac{\zeta}{\pi} \iint_{\Delta_{r}} \frac{v(z)}{z^{2}} d x d y, & \text { for } & |\zeta|=r,
\end{array}\right. \\
f[v](\zeta)=\frac{\zeta}{\pi} \iint_{\Delta_{r}} \sum_{k=-\infty}^{+\infty} \frac{v(z)}{z^{2}}\left[\frac{\zeta+r^{2 k} z}{\zeta-r^{2 k} z}-\frac{1+r^{2 k} z}{1-r^{2 k} z}\right] d x d y \\
\iint_{\Delta_{r}} v(z) g(z) d x d y=\frac{i}{2 \pi} \iint_{\Delta_{r}} \frac{v(z)}{z^{2}} d x d y \int_{|z|=r} z g^{*}(z) d z
\end{gathered}
$$

for all $g$ holomorphic in int $\Delta_{r}$ with $\iint_{\Delta_{r}}|g(z)| d x d y<\infty$.

Proof. The proof of (12) is presented with details in [6] and [2], the condition (13) is an immediate consequence of (5) and the definition of the class $N_{r}$. To get the condition (14) suppose that $g$ is holomorphic in int $\Delta_{r}$ with finite $L^{1}$ norm in $\Delta_{r}$. Then, by (12) and the generalized form of Green's formulae we get (14), where $g^{*}\left(r e^{i \theta}\right)=\lim _{\varrho \rightarrow r} g\left(\varrho e^{i \theta}\right)$, and $g^{*}\left(e^{i \theta}\right)=\lim _{\varrho \rightarrow 1} g\left(\varrho e^{i \theta}\right)$ which exists at almost all points of $\partial \Delta_{r}$ (cf. [5], p. 334) while $\iint_{\Delta_{r}}|g(z)| d x d y<\infty$.

Conversely, if (14) is satisfied, then applying it to

$$
g(z)=\frac{1}{\pi}(\zeta-z), \quad \zeta \in \partial \Delta_{r}
$$

(such functions are admissible) and using Corollary ([5], p. 335) we get (12). 


\section{On the function $\Phi$}

From (5) we obtain the explicit representation

$$
\Phi[v](\zeta)=-\frac{\zeta}{\pi} \iint_{\Delta_{r}} \sum_{k=-\infty}^{+\infty} \frac{\overline{v(z)}}{\bar{z}^{2}}\left[\frac{1+r^{2 k} \zeta \bar{z}}{1-r^{2 k} \zeta \bar{z}}-\frac{1+r^{2 k} \bar{z}}{1-r^{2 k} \bar{z}}\right] d x d y .
$$

By this formula we obtain a necessary condition for the holomorphic mapping $\Phi[v]$.

For this purpose we differentiate (16) three times:

$$
\Phi^{\prime \prime \prime}[v](\zeta)=-\frac{12}{\pi} \iint_{\Delta_{r}} \overline{v(z)} \sum_{k=-\infty}^{+\infty} \frac{r^{4 k}}{\left(1-r^{2 k} \zeta \bar{z}\right)^{4}} d x d y .
$$

Since $v$ is bounded $(|v| \leqq M)$ we obtain

$$
\left|\Phi^{\prime \prime \prime}[v](\zeta)\right| \leqq \frac{12 M}{\pi} \iint_{\Delta_{r}} \sum_{k=-\infty}^{+\infty} \frac{r^{4 k}}{\left|1-r^{2 k} \zeta \bar{z}\right|^{4}} d x d y .
$$

Changing the order of integration and summation we have for $r \leqq|\zeta| \leqq 1$

$$
\begin{aligned}
& \left|\Phi^{\prime \prime \prime}[v](\zeta)\right| \leqq \frac{12 M}{\pi} \sum_{k=-\infty}^{+\infty} \iint_{\Delta_{r}} \frac{r^{4 k}}{\left|1-r^{2 k} \zeta \bar{z}\right|^{4}} d x d y \\
= & \frac{12 M}{\pi} \sum_{k=-\infty}^{+\infty} r^{4 k} \int_{r}^{1} \varrho d \varrho \int_{|z|=\varrho} \frac{1}{\left(1-r^{2 k} \zeta \bar{z}\right)^{2}\left(1-r^{2 k} \zeta \bar{z}\right)^{2}} \frac{d z}{i z} \\
= & 12 M \sum_{k=-\infty}^{+\infty} r^{4 k}\left[\frac{1}{\left(1-r^{4 k}|\zeta|^{2}\right)^{2}}-\frac{r^{2}}{\left(1-r^{4 k+2}|\zeta|^{2}\right)^{2}}\right] .
\end{aligned}
$$

Let us rewrite the above inequality in the form

$$
\left|\Phi^{\prime \prime \prime}[v](\zeta)\right| \leqq 12 M\left[\frac{1}{\left(1-|\zeta|^{2}\right)^{2}}+\frac{r^{2}}{\left(r^{2}-|\zeta|^{2}\right)^{2}}+G(\zeta, r)\right],
$$

where $G(\zeta, r)$ denotes the series in (19) without these two terms. Since $G(\zeta, r)$ is bounded in $\Delta_{r}$, then by this and by the well-known Laurent theorem we can state

Theorem 2. The holomorphic function $\Phi[v]$ defined by (11) remains continuous on $|\zeta|=r$ and $|\zeta|=1$.

\section{Other properties of the class $N_{r}$}

Suppose that

$$
v\left(\varrho e^{i \theta}\right)=\sum_{n=-\infty}^{+\infty} \alpha_{n}(\varrho) e^{i n \theta}, \quad r<\varrho<1
$$

which is the Fourier series of $v$. Let $g$ be as in Theorem 1 for which

$$
g(z)=\sum_{k=-\infty}^{+\infty} a_{k} z^{k}=\sum_{k=-\infty}^{+\infty} a_{k} \varrho^{k} e^{i k \theta}, \quad z=\varrho e^{i \theta}, \quad r<\varrho<1
$$


is its Laurent series. Now, by the argument given in the proof of Theorem 1 , we may interpret (14) in terms of the coefficients $\alpha_{n}(\varrho)$ and $a_{k}, n, k=0, \pm 1, \pm 2, \ldots$. By this we have

$$
\begin{gathered}
\iint_{\Delta_{r}} v(z) g(z) d x d y=2 \pi \int_{r}^{1}\left\{\sum_{n=-\infty}^{+\infty} \alpha_{n}(\varrho) a_{-n} \varrho^{1-n}\right\} d \varrho \\
=2 \pi \sum_{n=-\infty}^{+\infty} a_{-n} \int_{r}^{1} \alpha_{n}(\varrho) \varrho^{1-n} d \varrho .
\end{gathered}
$$

For the right side of (14) we have

$$
\begin{gathered}
\frac{i}{2 \pi} \iint_{\Delta_{r}} \frac{v(z)}{z^{2}} d x d y \int_{|z|=\mathbf{r}} z g(z) d z \\
=-\frac{1}{2 \pi} \iint_{\Delta_{r}} \frac{v(z)}{z^{2}} d x d y \int_{|z|=r} z^{2} g(z) \frac{d z}{i z}=-2 \pi a_{-2} \int_{r}^{1} \frac{\alpha_{2}(\varrho)}{\varrho} d \varrho .
\end{gathered}
$$

Let$$
A_{n, k}=\int_{r}^{1} \alpha_{n}(\varrho) \varrho^{k+1} d \varrho,
$$

then by (23) and (24) the equality (14) can be expressed in the form

$$
\sum_{n=-\infty}^{+\infty} a_{-n} A_{n,-n}=-a_{-2} A_{2,-2} \text {. }
$$

Let $H(D)$ denote the Banach space of all holomorphic functions with finite $L^{1}$-norm in domain $D$. If $D_{1} \subset D_{2}$, then clearly $H\left(D_{1}\right) \supset H\left(D_{2}\right)$.

In the case of the unit disc it is easy to see that the unit disc can be replaced by an arbitrary simply connected region $D$. If $D_{1} \subset D_{2}$ and $v \in N\left(D_{1}\right)$, then $\tilde{v} \in N\left(D_{2}\right)$, where

$$
\tilde{v}(z)= \begin{cases}v(z), & z \in D_{1} \\ 0, & z \in D_{2} \backslash D_{1}\end{cases}
$$

Making use of (14) we see that the previous implication is also true in the case of a doubly connected domain.

These results have natural analogues in the case $r=0$, i.e., for mappings in the unit disc with an additional invariant point zero.

\section{References}

[1] AhLfors, L. V.: Some remarks on Teichmüller's space of Riemann surfaces. - Ann. of Math. 74, 1961, 171-191.

[2] LAWRYNowicz J., and J. KRZYŻ, Quasiconformal mappings in the plane: Parametrical methods. - Lecture Notes in Mathematics 978. Springer-Verlag, Berlin-HeidelbergNew York-Tokyo. 1983.

[3] ReICH, E.: An extremum problem for analytic functions with area norms. - Ann. Acad. Sci. Fenn. Ser. A I Math. 2, 1976, 429-445. 
[4] Reich, E., and K. StREBel: On quasiconformal mappings which keep the boundary points fixed. - Trans. Amer. Math. Soc. 138, 1969, 211-222.

[5] Rudin, W.: Real and complex analysis. - McGraw-Hill Book Company, New York-St. LouisSan Francisco-Toronto-London-Sydney, 1966.

[6] ZAJĄC, J.: The Ahlfors class $N$ and its connection with Teichmüller quasiconformal mappings of an annulus. - Ann. Univ. Mariae Curie-Skłodowska Sect. A 32, 1978, 155-162.

University Mariae Curie-Skłodowska Polish Academy of Sciences Institute of Mathematics 20-031 Lublin Institute of Mathematics Poland 90-136 Łódź

Poland

Received 29 May 1986 